osmotischen Messungen sich beim Studium der Elektroosmose an keramischen Diaphragmen gut bewährt haben, gibt uns die berechtigte Hoffnung, daß man den beschriebenen Apparat und die angeführte Methode mit Erfolg - vielleicht mit gewissen kleineren Anderungen — auch beim Studium der Elektroosmose an anderen porösen Materialen oder mit anderen Elektrolyten anwenden kann.

\section{Zusammenfassung.}

Auf Grund des vieljährigen Studiums beschreibt der Autor einen Apparat und eine Me- thode für elektroosmotische Messungen an keramischen Diaphragmen mit $\mathrm{KCl}$-Lösungen.

Auf Grund dieser experimentellen Studien der Elektroosmose kann man bestimmen:

1. Die gesamte Uberführung (d. i. sowohl die elektroosmotische als auch die elektrolytische Uberführung) der Elektrodenlösung in Litern per $1 \mathrm{~F}(=96490$ Coulomb).

2. Die relative Erhöhung der Leitfähigkeit der untersuchten Elektrodenlösung in Kapillarenräumen des Diaphragmas gegenüber der ,freien“ Elektrodenlösung. Der angegebene Apparat und die beschriebene Methode ergeben Meßresultate von sehr großer erreichbarer Genauigkeit.

\title{
Über kolloides Kupfer und alkoholisches Aluminiumoxyd-Gel.1)
}

\author{
Von Mike A. Miller. ${ }^{2}$ )
}

(Eingegangen am 2. August 1936.)

(Aus dem Chemischen Laboratorium der Universität Michigan, Ann Arbor, Michigan, USA.)

Für die Bereitung von Kupferhydroxyd- und Aluminiumoxyd-Solen und - Gelen $\left.^{3}\right)^{4}$ ) sind verschiedene Methoden angegeben worden. Viele dieser Methoden ergeben ein Produkt, welches unerwünschte Verunreinigungen enthält. Im Laufe gewisser von Kutzelnigg und Wagner ${ }^{5}$ ) beschriebener Experimente wurden einige Beobachtungen gemacht, welche in dieser Beziehung eine besondere Bedeutung haben. Kutzelnigg und Wagner stellten Aluminiumoxydgele her, indem sie (mittels verd. Alkalilösung gereinigtes) Aluminiummetall mit einer 2proz. Losung von Kupferchlorid in 95 proz. Alkohol reagieren ließen. Unter Entwicklung von Wasserstoff setzte sich auf dem Aluminiummetall Kupfer ab, wobei sich ein alkoholisches Aluminiumoxydgel bildete. Mit richtig eingesteliten Konzentrationen kann ein solches Gel frei von Kupferion erhalten werden, stets wird aber Chlorion enthalten sein.

1. Kolloides Kupferund Kupferhydroxyd.

Eine Scheibe aus ca. 99,5 proz. Aluminium von $2 \mathrm{~cm}$ Durchmesser und $1 \mathrm{~mm}$ Dicke wurde in verdünnter Alkalilauge gereinigt, dann mit einer großen Menge destilliertem Wasser und

1) Deutsch bearbeitet von R. Walter (Leipzig).

2) Aluminium Research Laboratories, Aluminium Company of America, New Kensington, Pennsylvania, USA.

3) L. S. Finch, J. physic. Chem. 18, 261 (1914).

$\left.{ }^{4}\right)$ Siehe z. B. H. B. We iser, J. physic. Chem. 27, 685 (1923); ,Hydrous Oxydes", Mc Graw-Hill Book Co. 1926, 29, 87-I01, 121.

5) A. Kutzelnigg und W. Wagner, Kolloid-Z. 64, 209 (1933). schließlich mit 95 proz. Athylalkohol gewaschen. Das so behandelte Aluminium wurde nun in eine mit Glasstöpsel versehene Weithalsflasche gegeben, die $30 \mathrm{ccm}$ einer zweiprozentigen Lösung von Kupferchlorid in 95 proz. Athylalkohol enthielt. Nach 72stündiger Reaktionsdauer wurde die Aluminiumscheibe mit dem lose anhaftenden Kupferfilm aus der Lösung entfernt und mit drei oder vier getrennten $10 \mathrm{ccm}-P$ ortionen von 95 proz. Athylalkohol durch Einweichen, Schütteln und Ausgießen gewaschen, wobei diese Portionen verworfen wurden. Die vierte oder fünfte 10-ccm-Portion ,,peptisiert" etwas von dem lose anhaftenden, fein verteilten Kupfer und wurde gesammelt.

Das resultierende alkoholische Sol erschien rötlichbraun im reflektierten Licht und grauviolett in durchscheinendem Licht. Um schwebende Kupferteilchen zu entfernen, wurde durch ein Schleicher \& Schüll-Filter (575 hart) filtriert. Die ultramikroskopische Prüfung ergab kolloide Kupferteilchen ähnlich jenen, die mittels der Bredig-Lichtbogenmethode erhalten werden. Die Durchschnittsgröße der so ultramikroskopisch festgestellten Teilchen war $0,8 \mu$.

Nachdem das alkoholische Kupfersol ungefähr einen Tag ungestört gestanden hatte, bildete sich ein feiner, schwarzer Niederschlag, welcher selbst bei kräftigem Schütteln nicht zerstört wurde. Innerhalb einiger Tage nach dieser Koagulation hatte der schwarze Niederschlag (wahrscheinlich wasserfreies Kupferoxyd und vielleicht etwas metallisches Kupfer) sich von selbst dispergiert und bildete ein bläuliches Sol, welches 
im Charakter mit hydratisiertem Kupferoxydsol identisch war. Die ultramikroskopische Untersuchung zeigte diffuse Lichtkegel statt der früheren hellen Lichtpunkte.

Sowohl das metallische Sol als auch das Oxydsol koagulierten bei Elektrolytzugabe, die letzteren jedoch weniger leicht als die ersteren und erst nach fortgesetztem Erwärmen. Gleiche Filtratvolumen jedes der beiden koagulierten Sole wurden mit demselben Gewicht wasserfreiem Kupfersulfat behandelt. Das Filtrat des koagulierten Oxydsols zeigte entschieden geringere Farbe als das Filtrat des koagulierten Metallsols.

Die Oxydation scheint aus der Wechselwirkung zwischen metallischem Kupfer (wahrscheinlich in irgendeiner aktivierten Form) und Wasser (oder Sauerstoff) hervorzugehen. Das Kupfer und das Kupferoxyd fällen sich offensichtlich gegenseitig durch Neutralisation der Ladungen. Nach Hydratisierung des Kupferoxyds durch Adsorption von Wasser resultiert das Kupferhydroxydsol in Athylalkohol.

\section{Alkoholisches Aluminiumoxyd gel.}

Mehrere Aluminiumscheiben, die gleich lange mit angesetztem Kupfer bedeckt waren [entsprechend dem Kutzelnigg - Wagn e r'schen Versuch $\left.^{6}\right)$ ] wurden sorgfältig in vielen frischen Bädern von 95 proz. Athylalkohol gewaschen, bis keine weitere Peptisation von lose anhaftenden Kupferteilchen mehr stattfand. In je eine von drei mit Glasstöpsel versehenen Weithalsflaschen, die $10 \mathrm{ccm}$ a) Leitfähigkeitswasser, b) absoluten Athylalkohol und c) 95 proz. Athylalkohol enthielten, wurde eine Scheibe Aluminium gelegt. Die Flaschen a) und b) zeigten während des begrenzten Zeitabschnitts der Beobachtung bis auf eine leichtflockige Aluminiumsuspension im Falle a) keine sichtbare Reaktion. Flasche c) jedoch zeigte die Bildung eines farblosen Aluminiumoxydgels um die kupferbedeckte Aluminiumscheibe herum in Form einer festen durchsichtigen Masse. Während dieses Vorganges entwickelte sich langsam Wasserstoff.

Um festzustellen, ob diese Reaktion auch vor sich geht, wenn keine Spuren von Elektrolyt vorhanden sind, wurde folgender Versuch durchgeführt: Sehr reiner Aluminiumdraht, welcher mit verdünnter Natronlauge gereinigt und mit einer großen Menge destilliertem Wasser gewaschen wurde, wurde unter 95 proz. Athylalkohol gefeilt und die Späne gesammelt. Diese wurden

$\left.{ }^{6}\right)$ Loc. cit. sofort in ein Reagensglas, welches 95 proz. Athylalkohol enthielt, gegeben. Angenähert die gleiche Menge reiner Kupferspäne wurde zugefügt. Innerhalb weniger Tage war das Gemisch der Aluminium- und Kupferteilchen von einer festen Schicht farblosen Aluminiumoxydgels umhüllt. Nach ungefähr zwei Wochen war das Aluminium größtenteils in Aluminiumoxydgel umgewandelt worden, während das Kupfer nicht angegriffen zu sein schien. Es wurde festgestellt, daB sich während dieses Vorgangs winzige Blasen von Wasserstoff bildeten.

\section{Besprechung der Ergebnisse.}

Die oben beschriebenen Reaktionen können stattfinden infolge der vielen elektrochemischen Elemente, welche durch den metallischen Kontakt des Aluminiums und Kupfers gebildet werden. Tammann und Ruhenbeck ${ }^{7}$ ) haben jedoch gezeigt, daß gewisse binäre Verbindungen von Aluminium in feuchter Luft zerfallen, während gewisse andere, einschließlich dem wohlbekannten $\mathrm{CuAl}_{2}$, dies nicht tun. Die Autoren folgern, daß diese Form des Zerfalls nicht elektrochemischer Natur ist. Anderseits ist darauf hingewiesen worden ${ }^{8}$ ), daß in gewissen lösungen das Aluminium gegenüber Kupfer ein Potential von einigen Zehntel Volt zeigen kann. Tammann und Boehme $\mathrm{e}^{9}$ ) haben auch gefunden, daß die Auflösungsgeschwindigkeit von Duraluminium in Salzsäure durch niedergeschlagene $\mathrm{CuAl}_{2}$-Teilchen entschieden beschleunigt wird. Diese Teilchen bilden eine Reihe von galvanischen Zellen mit dem basischen Metall.

Nach den wenigen vorliegenden anderweitigen Beobachtungen kann nicht mit Bestimmtheit gesagt werden, ob die in dieser Mitteilung beschriebene Reaktion eine rein chemische Zersetzung des Wassers oder ein elektrochemischer Vorgang ist. Auf jeden Fall müßte die beschleunigende Wirkung des Kupfers in Athylalkohol und der poröse Charakter des Aluminiumoxyds, welches gebildet wird, bei einer Erklärung des Vorgangs in Erwägung gezogen werden.

\section{Zusammenfassung.}

1. Es wurde festgestellt, daß das feinverteilte Kupfer, welches auf Aluminium aus einer Kupfer-

7) Tammann und Ruhenbeck, Z. anorg. allg. Chem. 223, 288 (1935).

8) Siehe z. B. J. D. Edwards und C. S. Taylor,

Trans Amer. Elektrochem. Soc. 56, 27 (1929).

9) Tammann und Boehme, Z. anorg. allg. Chem. 226, 82 (1935). 
chloridlösung abgeschieden wird, peptisiert werden kann und ein Sol mit einer durchschnittlichen Teilchengröße der Kupferpartikelchen von $0,8 \mu$ bildet.

2. Ein derartiges Sol scheint sich in Athyl- alkohol von selbst in Kupferhydroxyd zu verwandeln.

3. Aluminium und Kupfer in metallischem Kontakt bilden in 95 proz. Athylalkohol ein Aluminiumoxydgel.

\section{Über Humussäuren.}

\section{Mitteilung.}

Von Walter Scheele und Leonore Steinke.

(Aus dem Chemischen Institut der Universität Greifswald.)

(Eingegangen am 18, Okțober 1936.)

\section{Einleitung und Problemstellung.}

Humussäuren sind dunkelbraune organische Naturstoffe mit ausgesprochen kolloider Beschaffenheit und entstehen wahrscheinlich durch bakteriellen Abbau des Lignins in allerdings im einzelnen noch nicht ganz durchsichtiger Weise. Sie stellen die relativ beständigen Endprodukte der Humifizierung vor und spielen als solche eine große Rolle im Haushalt der Natur. Wegen der Bedeutung dieser Stoffe und wegen ihres auBerordentlich verbreiteten Vorkommens hat man sich seit langer Zeit mit ihrer Chemie befaßt ${ }^{1}$ ). Mit den Methoden, die die organische Experimentalchemie bietet, ist es bis heute nicht gelungen, die Konstitution dieser Verbindungen bis in alle Einzelheiten festzulegen. Genauer unterrichtet sind wir lediglich über einige konstituierende Gruppen im Humusmolekül ${ }^{2}$ ). Im übrigen aber bestehen über den inneren Aufbau der Humussubstanz nur Vermutungen.

Die Konstitutionsermittlung der Humussäuren ist zweifelsohne mit prinzipiellen Schwierigkeiten verbunden; denn die Humussäuren sind kolloide Stoffe und zeigen je nach Art ihrer Gewinnung und je nach dem Material, aus dem sie gewonnen wurden, verschiedene Elementarzusammensetzung und verschiedenes Verhalten gegenüber Lösungsmitteln, Umstände, die dazu geführt haben, in dem Humus ein Stoffgemenge zu erblicken, für dessen Trennung in einzelne definierte Produkte wir zunächst keine Möglichkeit sehen.

Uns erschien es aussichtsvoll, systematisch mit einigen physikalisch-chemischen Methoden an die Bearbeitung des Problems heranzugehen,

1) W. Fuchs, Die Chemie der Kohle (Berlin 1931); Kolloid-Z. 52, 248 4. 350 (1930); G. Stadnik off, Neuere Torfchemie (Dresden a. Leipzig 1930); Sven Odén, Die Huminsäuren (Dresden u. Leipzig 1919).

2) W.Fuchs, Die Chemie der Kohle (Berlin 1931). wobei wir als nächstes Ziel eine Analyse des molekularen Zustandes natürlicher Humusstoffe ins Auge faßten, um auf diese Weise gegebenenfalls genauere Aussagen darüber machen zu können, welcher Art das Stoffgemenge Humus ist.

In unseren Untersuchungen über Humussäuren ${ }^{3}$ ) haben wir bisher besonders mit Hilfe einiger konduktometrischer Titrationsverfahren die Salzbildung verschiedener Humussäuren quantitativ gemessen. Dabei wurde die Bestimmung des Aquivalentgewichtes von Humussäuren methodisch auf eine exakte Grundlage gestellt. Zwei Methoden wurden genauer beschrieben, die die Bestimmung des Aquivalentgewichtes mit einer Genauigkeit von 2,5 Proz. ermöglichen. Die gemessenen Aquivalentgewichte dienten uns zunächst zur Charakterisierung unserer Präparate. Sie wurden in allen bislang untersuchten Fällen kleiner gefunden als von Odén ${ }^{4}$ ) und dürften nach unseren neueren Erfahrungen bei ganz reinen und wasserfreien Präparaten um 200 liegen. Humussäuren, Huminsäuren und Hymatomelansäuren zeigten annähernd gleiche Äquivalentgewichte.

Im weiteren Verlaufe unserer Arbeiten haben wir Molekulargewichtsbestimmungen von Humussäuren durchgeführt. Die vorliegende Abhandlung bringt die Ergebnisse solcher Untersuchungen an einer Humussäure aus Kasseler Braun.

Molekulargewichte von Humussäuren wurden erstmalig von $\mathrm{Odé} \mathrm{n}^{5}$ ) mitgeteilt. Sie wurden von ihm nicht direkt gemessen, sondern aus einem Aquivalentgewicht von 340 unter der Annahme, daß die Humussäure eine vierbasische Säure ist, errechnet. Danach hätten die Humussäuren ein Molgewicht, das zwischen 1300 und 1400 liegt. Aus der beobachteten Siedepunktserhöhung alko-

3) W. Scheele und Mitarbeiter, Kolloid-Z. 72, 301 (1935); 73, 84; 75, 73 (1936).

4) Siehe Fußnote 1 .

5) Siehe Fußnote 1. 